\title{
The burden of amyloid light chain amyloidosis on health-related quality of life
}

\author{
Martha Bayliss', Kristen L. McCausland ${ }^{1 *}$, Spencer D. Guthrie ${ }^{2}$ and Michelle K. White ${ }^{1}$
}

\begin{abstract}
Background: Light chain (AL) amyloidosis is a rare disease characterized by misfolded amyloid protein deposits in tissues and vital organs, and little is known about the burden of AL amyloidosis on health-related quality of life. This study aimed to quantify the burden of AL amyloidosis in terms of health-related quality of life in a diverse, community-based sample of AL amyloidosis patients.

Results: The SF-36v2 ${ }^{\circledR}$ Health Survey (SF-36v2), a widely used generic measure of health-related quality of life (using physical and mental summary scales and subscales assessing eight aspects of functioning and well-being), was administered as an online survey of AL amyloidosis patients with AL amyloidosis (ClinicalTrials.gov, NCT02574676; $n=341$ ). Compared with adjusted general population sample norms, health-related quality of life of AL amyloidosis patients was significantly worse across all SF-36v2 scales and summary measures based on analysis of variance $(p<0.05$ for all). The largest decrement in AL amyloidosis patients was related to General Health $(\Delta=9.7 ; p<0.001)$. With the exception of Bodily Pain and Mental Health, differences were also clinically meaningful based on established clinically minimal important differences. The burden of AL amyloidosis overall and in key subgroups tended to be greater on physical health than on mental health. Stratified analyses indicated additional burden among patients with recently diagnosed disease and those with cardiac involvement than among their respective counterparts.

Conclusion: Understanding the burden of AL amyloidosis highlights the unmet need for treatment, helps physicians identify ancillary treatments and services geared towards improving patients' functioning, well-being, and overall health-related quality of life. These findings also help to support the use of health-related quality of life end points as important outcome measures in current and future treatment studies.
\end{abstract}

Trial registration: ClinicalTrials.gov, NCT02574676. Registered October 5, 2015.

Keywords: Amyloidosis, Burden, Quality of life, Rare disease, SF-36

\section{Background}

Systemic amyloidoses are a group of rare diseases characterized by abnormally folded protein (amyloid) deposits in body tissue and organs. Accumulation of these insoluble amyloid deposits can lead to organ toxicity, irreversible organ damage, dysfunction, and death [1]. Amyloidosis subtypes are defined by their fibril composition and precursor proteins. In amyloid light chain (AL) amyloidosis, the amyloid deposits are created by immunoglobulin light chain proteins produced by abnormal monoclonal plasma cells [2]. The estimated incidence of AL amyloidosis is 8 to 12 cases per million person-years [3, 4].

\footnotetext{
* Correspondence: kmccausland@qualitymetric.com

'Optum, 24 Albion Road, Lincoln, RI 02865, USA

Full list of author information is available at the end of the article
}

$\mathrm{AL}$ amyloidosis is a complex disease with a variety of clinical manifestations, nonspecific symptoms that are associated with a wide range of diseases, and a high-case fatality rate-factors that can contribute to challenges in early diagnosis [5]. Symptoms and complications depend on the number and types of organ systems involved and the duration of time between symptom onset and treatment. Although estimates vary, approximately $70 \%$ of patients have cardiac involvement and $68 \%$ have multiple organ involvement at diagnosis [6]. Damage to multiple organs and cardiac impairment are predictive of decreased survival rates and poor disease outcomes; the most common cause of death is cardiac complication [7]. 
Prognosis for patients with AL amyloidosis is improved by early diagnosis and treatment. No treatments have been approved by either the United States Food and Drug Administration (FDA) or the European Medicines Agency (EMA), leaving substantial unmet need for patients. Typical treatments, which are adapted from regimens used to treat multiple myeloma, include chemotherapy, stem cell transplantation (SCT), and immunomodulatory drugs. These treatments target plasma cells and aim to reduce the production of amyloid-forming light chains. Existing regimens can be associated with significant tolerability problems, including treatment-emergent symptoms $[8,9]$.

Understanding the patient experience, including physical and mental aspects of health-related quality of life (HRQoL), can help to characterize burden of disease. Qualitative research indicates that patients with $\mathrm{AL}$ amyloidosis experience substantial burden that can lead to impairment in daily functioning [10, 11]. Furthermore, AL amyloidosis can lead to anxiety, frustration, and depression as patients grapple with the gravity and rarity of their condition [10, 12]. Although limited, quantitative studies provide evidence of deficits in both physical and mental aspects of HRQoL $[13,14]$.

Given the absence of a disease-specific measure of HRQoL for AL amyloidosis and the disease heterogeneity, a general health status measure, such as the SF-36v2 Health Survey ${ }^{\oplus}$ (SF-36v2), is well suited for assessing HRQoL in this population [15]. The SF-36v2 is a widely used generic measure of HRQoL and the most common patient-reported outcome end point in clinical trials [16-18]. Generic measures of HRQoL allow for comparisons with the general population and other disease populations, which can provide context for rare diseases $[19,20]$.

Studies examining the impact of AL amyloidosis on HRQoL, particularly among patient subgroups, are scant. Accordingly, this study used the SF-36v2 to compare the HRQoL burden of AL amyloidosis patients and two key subgroups hypothesized to have greater disease severity with that observed in the US general population (GP).

\section{Methods}

\section{Sample/study procedures}

The study is a cross-sectional analysis of baseline data $(n=341)$ taken from the AL Amyloidosis Patient Health-Related Quality of Life Study (ClinicalTrials.gov; NCT02574676), an online noninterventional, longitudinal study of patients with AL amyloidosis. This study was approved by the New England Institutional Review Board. Recruitment took place between October and December 2015, with scripted messages posted on patient advocacy group Web sites and social media sites and in membership e-mails. These messages provided a hyperlink to an electronic informed consent form and screening questions. Potential participants were eligible to participate if they were $\geq 18$ years of age, received a diagnosis of AL amyloidosis from a physician, and were willing and able to complete four online surveys over the course of 12 months. Those who met inclusion criteria were automatically directed to the baseline survey.

\section{Study measures}

Several items capturing basic demographic, disease, and treatment characteristics were used to describe the sample. Dates of diagnosis and survey completion were used to calculate the time since each patient received the AL amyloidosis diagnosis. Specific organ involvement was measured with a six-item checklist of organs or systems commonly affected by AL amyloidosis, including heart (cardiac), kidney, liver, nervous system, and gastrointestinal system. Responses to these items were summed to generate a composite measure of the total number of organs affected. Hematologic response (HR), based on the assessments of serum-free light chains, is often used as a measure of treatment efficacy for AL amyloidosis [21]. In this study, a proxy measure of this clinical information was developed by asking patients to describe their most recent HR status in terms of the following: (1) no response to treatment, (2) partial HR or partial remission, (3) complete HR or complete remission, or (4) I do not know.

The SF-36v2 (with 4-week recall) was used to measure general HRQoL burden in eight dimensions of functional health and well-being: Physical Functioning (PF), Role-Physical (RP; role limitations due to physical problems), Bodily Pain (BP), General Health Perceptions $(\mathrm{GH})$, Vitality (VT), Social Functioning (SF), RoleEmotional (RE; role limitations due to emotional problems), and Mental Health $(\mathrm{MH})$. Item responses were used to calculate scale scores for each of the eight dimensions, and summary scores (Physical Component Summary [PCS] and Mental Component Summary [MCS]) were computed from weighted scores from the eight scales. All scores were calculated using a scoring algorithm that yields standardized $\mathrm{T}$ scores for a nationally representative sample of US adults [15]. Higher SF36v2 scores represent better health. Previously reported minimal clinically important differences (MCIDs) for each of the eight scales, PCS, and MCS were used to interpret whether statistically significant differences were also clinically meaningful [15].

Two other survey items were used to describe burden in terms of current disease status or severity: a global assessment of functioning on a scale of 0 to 100 (higher scores mean better functioning) and the Patient Global Impression-Severity scale (PGI-S). The PGI-S was used to assess the severity of $\mathrm{AL}$ amyloidosis in the past month on a five-point scale (not severe at all, mild, moderate, severe, very severe) [22]. 


\section{Statistical analyses}

Demographics, disease characteristics, and HRQoL were compared in stratified analyses of two clinically relevant subgroups: diagnosed $<12$ months ago versus diagnosed $\geq 12$ months ago; cardiac involvement versus no cardiac involvement. Unadjusted differences between the subgroups were examined using chi-square and WilcoxonMann-Whitney tests, as appropriate.

SF-36v2 scores for all patients and the key clinical subgroups were compared with those from the GP. Normative data were drawn from the QualityMetric 2009 Norming Study $(N=4040)$, a cross-sectional online study conducted between June and October 2009. The methodology for the GP has been described [15]. Participants were recruited from the Knowledge Panel ${ }^{\circ}$, a probability sample of US households [23], to complete an online survey that included the SF-36v2.

Data from the GP were adjusted to the age and gender distribution of the AL amyloidosis sample using separate least squares multiple regression models for each SF$36 \mathrm{v} 2$ scale and summary score. Analysis of variance methods were used to test for significant differences between scores obtained from the AL amyloidosis sample and the adjusted norms.

All analyses were performed using SAS version 9.2 (SAS Institute Inc, Cary, NC).

\section{Results}

\section{Sample characteristics}

Sample characteristics are reported in Table 1. The average age of the patients was 61 years. Slightly more women $(52.9 \%)$ than men $(47.1 \%)$ participated. Most patients were white $(89.1 \%)$, well educated $(61.2 \%$ had at least a 4-year college degree), and married (82.1\%). Approximately $43 \%$ of patients were diagnosed $>1$ year after the onset of symptoms. Time since diagnosis varied from 1 month to 28 years (median, 3.5 years). Nearly 23\% of the sample reported a history of multiple myeloma. There was a broad representation of affected organs; the largest percentage of patients had cardiac (52.2\%) and/or kidney (62.8\%) involvement. In approximately $46.0 \%$ of patients, three or more organs were affected by $\mathrm{AL}$ amyloidosis. At the time of data collection, most patients who had received at least one treatment series had experienced some type of response to treatment (39.3\% partial HR, 43.6\% complete HR).

Patient demographics did not differ significantly by time since diagnosis or cardiac involvement (data not shown). Patients diagnosed $<12$ months ago differed from those who received their diagnoses $\geq 12$ months ago on several key aspects of disease and treatment (Table 2). Given their shorter duration of disease, greater proportions of patients diagnosed $<12$ months ago reported not having started treatment or having received only one treatment series to date. They were also less likely to have undergone SCT and less likely to have achieved complete HR than those with longer disease duration. Patients with cardiac involvement did not differ significantly from those without cardiac involvement by HR or history of SCT.

\section{The humanistic burden of AL amyloidosis}

Compared with age- and gender-adjusted GP norms, patients with AL amyloidosis had worse HRQoL, as demonstrated by their scores on all eight SF-36v2 scales and summary scores ( $p<0.05$ for all) (Fig. 1). All differences, with the exception of $\mathrm{BP}$ and $\mathrm{MH}$, exceeded the established MCIDs, indicating that these deficits are clinically meaningful as well as statistically significant. The largest differences were related predominantly to physical health status. Compared with the GP, the largest observed differences were related to $\mathrm{GH}(\Delta=9.7$; Cohen's $d,-0.65$; $p<0.001)$ and RP $(\Delta=7.1$; Cohen's $d,-0.49 ; p<0.001)$, with corresponding decrements in PCS $(\Delta=5.9$; Cohen's $d,-0.44 ; p<0.001)$. Significant decrements were also seen for scales assessing mental health status, such as SF $(\Delta=6.0$; Cohen's $d,-0.40 ; p<0.001)$ and VT $(\Delta=5.8$; Cohen's $d,-0.39 ; p<0.001)$, and a corresponding deficit was seen for the MCS $(\Delta=3.7$; Cohen's $d$, $-0.25 ; p<0.001)$.

Overall SF-36v2 scores and other HRQoL measures are reported in Table 3. The average global assessment of functioning score was 70.2 (SD, 23.7). Based on responses to the PGI-S, slightly more than one-third of all patients described the severity of their condition as moderate to very severe (34.0\%).

\section{The humanistic burden among patients with recently diagnosed AL amyloidosis}

Patients diagnosed $<12$ months earlier exhibited large decrements in all eight SF-36v2 scale scores, PCS, and MCS ( $p<0.05$ for all) compared with the GP (Fig. 2). Consistent with patterns observed in the overall sample, the burden among recently diagnosed patients was greater for physical health status than mental health status. The greatest HRQoL decrement among these patients was in RP $(\Delta=12.3$; Cohen's $d,-1.01 ; p<0.001)$, with other large decrements in $\mathrm{GH}(\Delta=10.2$; Cohen's $d,-0.81$; $p<0.001)$ and SF $(\Delta=10.8$; Cohen's $d,-0.85 ; p<0.001)$.

Deficits in HRQoL among AL amyloidosis patients with recent diagnoses often exceeded those among patients diagnosed $\geq 12$ months ago (Table 4). Patients with recent diagnoses reported worse scores on PF, RP, VT, SF, RE, MH, and MCS than did those diagnosed $\geq 12$ months ago $(p<0.05$ for all). Furthermore, all differences exceeded the established MCIDs for these scales/scores. There were no significant differences for 
Table 1 Demographic, Disease, and Treatment Characteristics of Study Participants

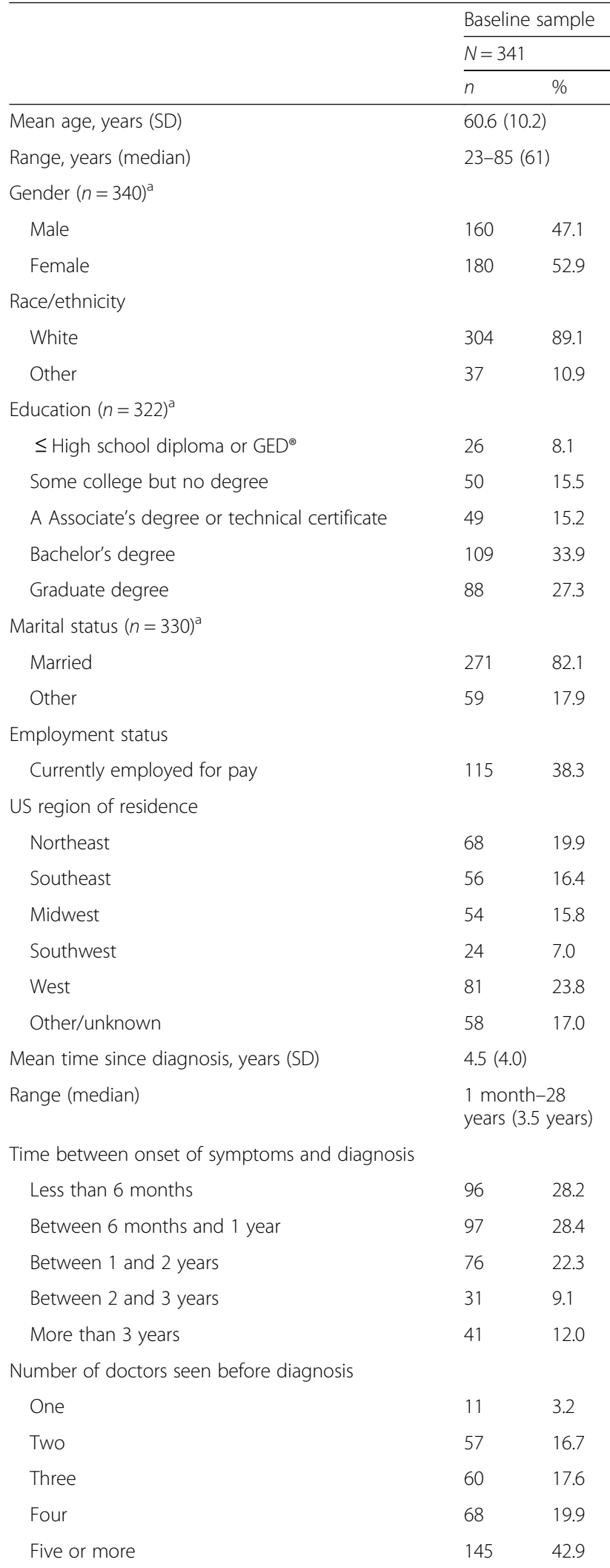

Table 1 Demographic, Disease, and Treatment Characteristics of Study Participants (Continued)

\begin{tabular}{|c|c|c|}
\hline \multicolumn{3}{|l|}{ Organs/systems impacted ${ }^{b}$} \\
\hline Heart (cardiac) & 178 & 52.2 \\
\hline Kidney & 214 & 62.8 \\
\hline Liver & 49 & 14.4 \\
\hline Nervous system & 126 & 37.0 \\
\hline Gastrointestinal & 148 & 43.4 \\
\hline Other & 117 & 34.3 \\
\hline \multicolumn{3}{|l|}{ Number of organs involved } \\
\hline One & 95 & 27.9 \\
\hline Two & 89 & 26.1 \\
\hline Three or more & 157 & 46.0 \\
\hline History of multiple myeloma & 71 & 22.8 \\
\hline \multicolumn{3}{|l|}{ No. treatment series received } \\
\hline None & 20 & 5.9 \\
\hline One & 70 & 20.5 \\
\hline Two & 76 & 22.3 \\
\hline Three & 61 & 17.9 \\
\hline Four & 25 & 7.3 \\
\hline Five or more & 89 & 26.1 \\
\hline Ever underwent stem cell transplantation & 180 & 52.9 \\
\hline \multicolumn{3}{|l|}{ Most recent hematologic response status $(n=321)^{c}$} \\
\hline No response to treatment & 20 & 6.2 \\
\hline Partial hematologic response or partial remission & 126 & 39.3 \\
\hline $\begin{array}{l}\text { Complete hematologic response or complete } \\
\text { remission }\end{array}$ & 140 & 43.6 \\
\hline I do not know & 35 & 10.9 \\
\hline
\end{tabular}

GED, General Educational Development; SD, standard deviation ${ }^{\mathrm{a}}$ Frequencies less than 341 are due to missing data; Percentages based on available data

${ }^{\mathrm{b}}$ Multiple response options allowed

${ }^{c}$ Frequencies and percentages are based on the 321 patients who received at least one treatment series

$\mathrm{BP}, \mathrm{GH}$, and PCS, though both groups of patients were severely impaired in these areas.

Responses to the global assessment of functioning and the PGI-S reinforced the finding of added burden among patients with recent diagnoses (Table 4). The mean global assessment of functioning score was approximately $22 \%$ greater for those with diagnoses made $\geq 12$ months ago than for those with more recent diagnoses (72.2 vs 59.1 , respectively; $p<0.05)$, indicating better functioning among those whose disease was diagnosed for a longer period of time. A greater proportion of patients with recent diagnoses described the severity of their disease as moderate to very severe (55.7\%) than did patients diagnosed $\geq 12$ months ago (30.0\%) $(p<0.001)$. 
Table 2 Disease and Treatment Characteristics by Time since Diagnosis and Cardiac Involvement

\begin{tabular}{|c|c|c|c|c|c|c|c|c|c|c|}
\hline & \multicolumn{4}{|c|}{ Time since diagnosis } & \multirow[b]{3}{*}{$P$ value } & \multicolumn{4}{|c|}{ Cardiac involvement } & \multirow[b]{3}{*}{$P$ value } \\
\hline & \multicolumn{2}{|c|}{$\begin{array}{l}<12 \text { months } \\
n=52\end{array}$} & \multicolumn{2}{|c|}{$\begin{array}{l}\geq 12 \text { months } \\
n=289\end{array}$} & & \multicolumn{2}{|c|}{$\begin{array}{l}\text { No } \\
n=163\end{array}$} & \multicolumn{2}{|c|}{$\begin{array}{l}\text { Yes } \\
n=178\end{array}$} & \\
\hline & $n$ & $\%$ & $n$ & $\%$ & & $n$ & $\%$ & $n$ & $\%$ & \\
\hline No. organs involved & & & & & 0.047 & & & & & $<0.001$ \\
\hline One & 20 & 38.5 & 75 & 26.0 & & 71 & 43.6 & 24 & 13.5 & \\
\hline Two & 16 & 30.8 & 73 & 25.3 & & 42 & 25.8 & 47 & 26.4 & \\
\hline Three or more & 16 & 30.8 & 141 & 48.8 & & 50 & 30.7 & 107 & 60.1 & \\
\hline No. treatment series received & & & & & & & & & & 0.889 \\
\hline None & 8 & 15.4 & 12 & 4.2 & $<0.001$ & 12 & 7.4 & 8 & 4.5 & \\
\hline One & 16 & 30.8 & 54 & 18.7 & & 32 & 19.6 & 38 & 21.3 & \\
\hline Two & 5 & 9.6 & 71 & 24.6 & & 34 & 20.9 & 42 & 23.6 & \\
\hline Three & 4 & 7.7 & 57 & 19.7 & & 29 & 17.8 & 32 & 18.0 & \\
\hline Four & 5 & 9.6 & 20 & 6.9 & & 12 & 7.4 & 13 & 7.3 & \\
\hline Five or more & 14 & 26.9 & 75 & 26.0 & & 44 & 27.0 & 45 & 25.3 & \\
\hline Ever underwent stem cell transplantation (\% yes) & 5 & 9.8 & 175 & 60.6 & $<0.001$ & 89 & 54.6 & 91 & 51.4 & 0.556 \\
\hline Most recent hematologic response status ${ }^{a}$ & & & & & $<0.008$ & & & & & 0.135 \\
\hline No response to treatment & 5 & 11.6 & 15 & 5.4 & & 9 & 6.0 & 11 & 6.5 & \\
\hline Partial hematologic response or partial remission & 19 & 44.2 & 107 & 38.5 & & 62 & 41.1 & 64 & 37.7 & \\
\hline Complete hematologic response or complete remission & 10 & 23.3 & 130 & 46.8 & & 58 & 38.4 & 82 & 48.2 & \\
\hline I do not know & 9 & 20.9 & 26 & 9.4 & & 22 & 14.6 & 13 & 7.7 & \\
\hline
\end{tabular}

${ }^{a}$ Frequencies and percentages are based on the 321 patients who received at least one treatment series

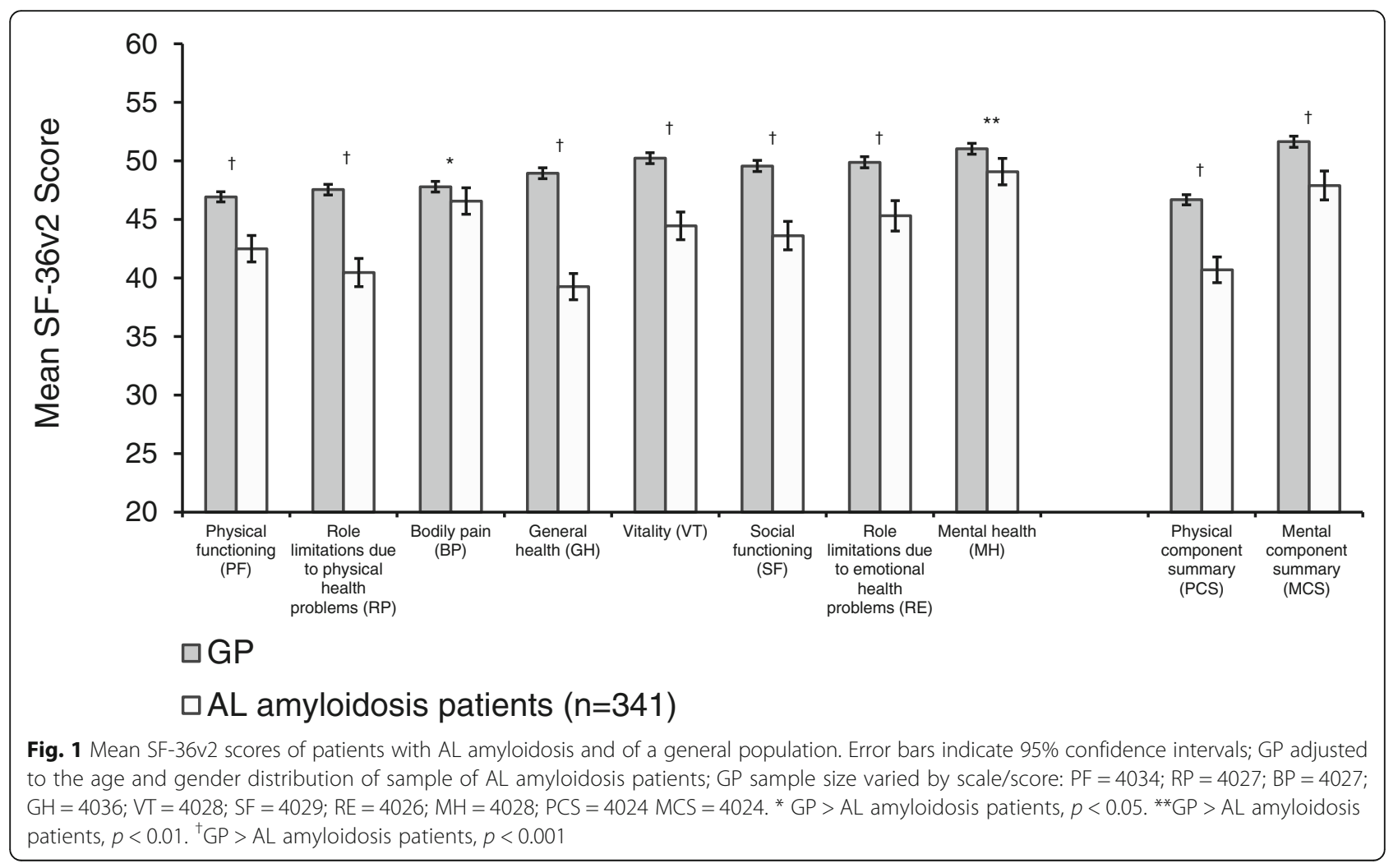


Table 3 Health-related quality of life among AL Amyloidosis patients $(n=341)$

\begin{tabular}{|c|c|c|}
\hline & \multicolumn{2}{|c|}{ AL amyloidosis patients $N=341$} \\
\hline & \multicolumn{2}{|l|}{ Mean (SD) } \\
\hline \multicolumn{3}{|l|}{ SF-36v2 norm-based scales ${ }^{a}$} \\
\hline Physical functioning (PF) & \multicolumn{2}{|l|}{$42.5(10.6)$} \\
\hline Role physical (RP) & \multicolumn{2}{|l|}{$40.5(11.3)$} \\
\hline Bodily pain (BP) & \multicolumn{2}{|l|}{$46.6(10.6)$} \\
\hline General health (GH) & \multicolumn{2}{|l|}{$39.3(10.6)$} \\
\hline Vitality (VT) & \multicolumn{2}{|l|}{$44.5(11.1)$} \\
\hline Social functioning (SF) & \multicolumn{2}{|l|}{$43.6(11.5)$} \\
\hline Role emotional (RE) & \multicolumn{2}{|l|}{$45.3(12.3)$} \\
\hline Mental health $(\mathrm{MH})$ & \multicolumn{2}{|l|}{$49.1(10.6)$} \\
\hline \multicolumn{3}{|l|}{ SF-36v2 Summary Scores } \\
\hline Physical Component Summary (PCS) & \multicolumn{2}{|l|}{$40.7(10.3)$} \\
\hline Mental Component Summary (MCS) & \multicolumn{2}{|l|}{$47.9(11.6)$} \\
\hline Global assessment of functioning & \multicolumn{2}{|l|}{$70.2(23.7)$} \\
\hline Patient Global Impression-Severity Scale (PGI-S) & $n$ & $\%$ \\
\hline Not severe at all & 133 & 39.0 \\
\hline Mild & 92 & 27.0 \\
\hline Moderate & 81 & 23.8 \\
\hline Severe & 23 & 6.7 \\
\hline Very severe & 12 & 3.5 \\
\hline
\end{tabular}

The humanistic burden among AL amyloidosis patients with cardiac involvement

Compared with the GP, patients with cardiac involvement also reported large decrements on all eight SF$36 \mathrm{v} 2$ scales and summary scores ( $p<0.05$ for all) (Fig. 3). With the exception of $\mathrm{BP}$ and $\mathrm{MH}$, all differences exceeded established MCIDs. Similar to the finding in the overall sample, the largest deficits were for scales that contribute to physical health status (ie, PF, RP, and $\mathrm{GH})$. Similarly, AL amyloidosis patients with cardiac involvement had significantly lower PCS scores than the adjusted GP $(p<0.001)$.

Results indicate that AL amyloidosis with cardiac involvement is associated with greater physical impairment than with non-cardiac organ involvement (Table 4). Mean SF-36v2 scores for patients with cardiac involvement were significantly lower for patients without cardiac involvement for three of the four physical scales (PF, RP, and GH; $p<0.05$ for all) and subsequently for PCS $(p<0.001)$. No significant differences were observed for $\mathrm{MH}$ scores or the MCS.

Responses on the PGI-S and global assessment of functioning items differed by presence of cardiac involvement (Table 4). Patients with cardiac involvement were half as likely as those without cardiac involvement to classify their condition as "not severe at all" $(27.5 \%$ vs $51.5 \%$, respectively). A significantly greater proportion of

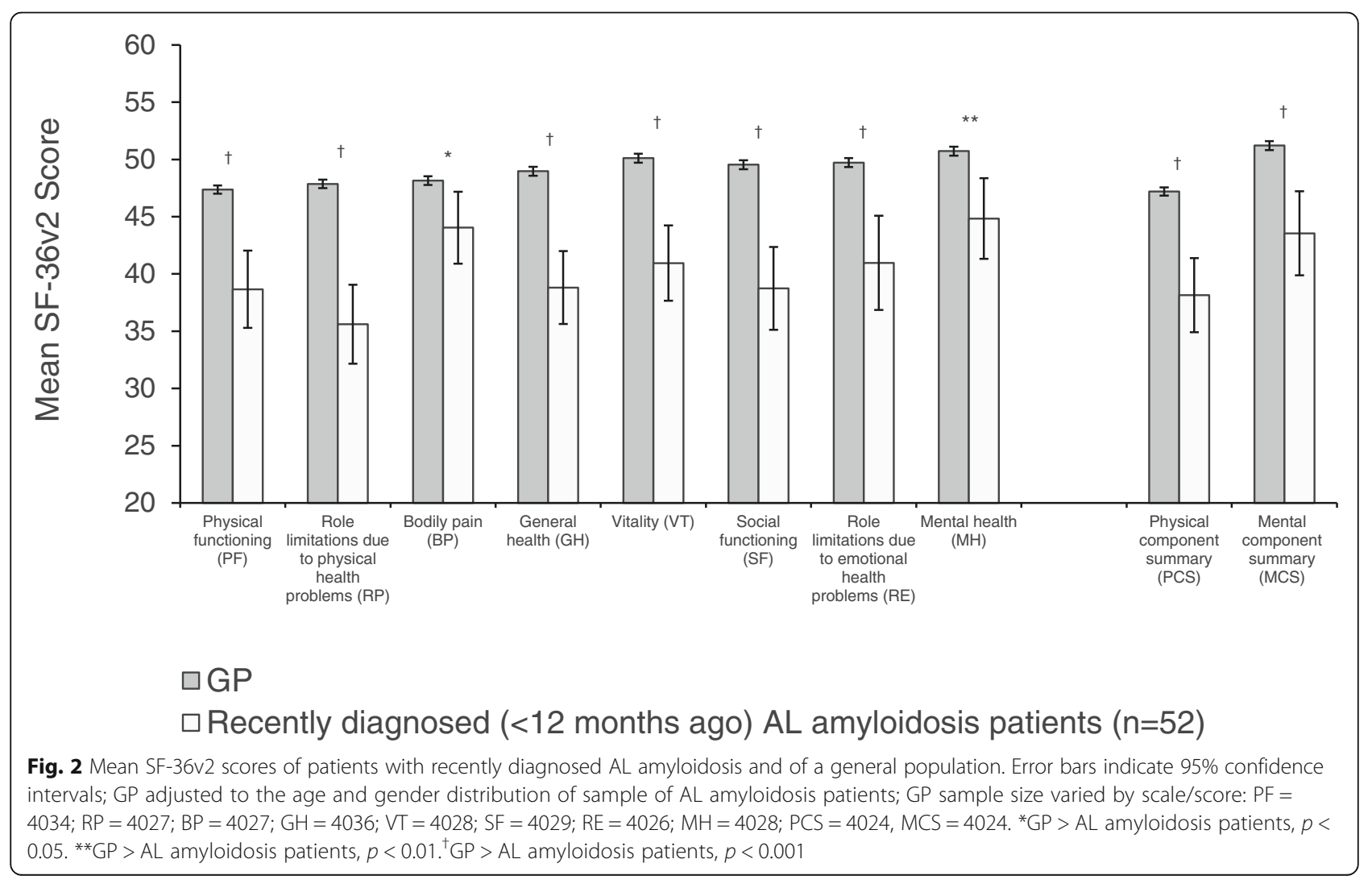


Table 4 Health-Related Quality of Life by Time since Diagnosis and Cardiac Involvement

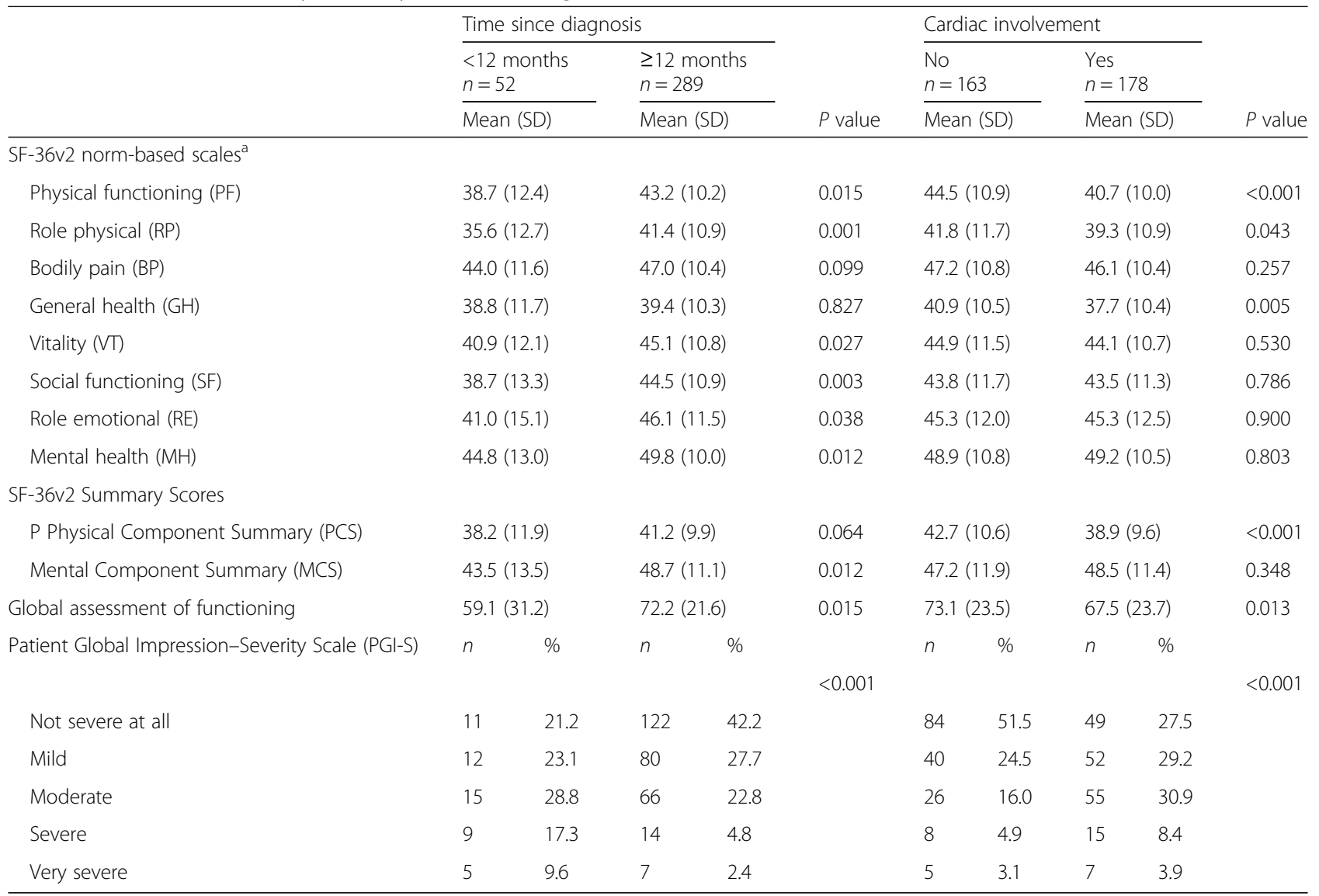

${ }^{a}$ SF-36v2, SF-36v2 Health Survey

patients with cardiac involvement than without it described the severity of their disease as moderate to very severe $(43.2 \%$ vs $24.0 \%$, respectively) $(p<0.001)$.

\section{Discussion}

These results indicate that AL amyloidosis patients have broad HRQoL deficits relative to a general population. Decrements in physical and mental functioning were statistically significant and often exceeded thresholds for clinically meaningful differences. The largest effects were observed in aspects related to physical functioning and general well-being. For instance, $\mathrm{GH}$ and $\mathrm{RP}$ were among the greatest deficits observed overall and in each key subgroup.

As expected, there was greater impairment in patients with recent diagnoses and those with cardiac involvement. Cardiac involvement in AL amyloidosis can lead to complications such as cardiomyopathy and heart failure. Although there are no other known studies that report HRQoL specifically in AL amyloidosis patients with cardiac involvement, our findings are congruent with assessments in populations with non-amyloidosis cardiomyopathy and heart failure [24].
This is the first study to characterize HRQoL in a community-based sample of AL amyloidosis patients and to further document HRQoL specifically in cardiac AL amyloidosis patients. Previous HRQoL-related studies in AL amyloidosis used older versions of the SF-36, relied on clinic-based samples, and predated many of the newer drugs used to treat AL amyloidosis [13, 14]. By partnering with patient advocacy groups, we were able to overcome some of the challenges of sample accrual and data collection often experienced in studies of rare diseases. Since recruitment occurred outside of the clinic setting, we were able to obtain a diverse sample of patients that included patients with recent diagnoses and long-term survivors. Rather than having patients complete a time-intensive survey in a clinic setting, the centralized, online mode of data collection used in this study allowed patients to complete the survey on their own time and from any location, which facilitated participation.

Despite the benefits of our recruitment and data collection strategies, several limitations are worth noting. All measures in this study relied on self-report. Although some measures were meant to be subjective (ie, 


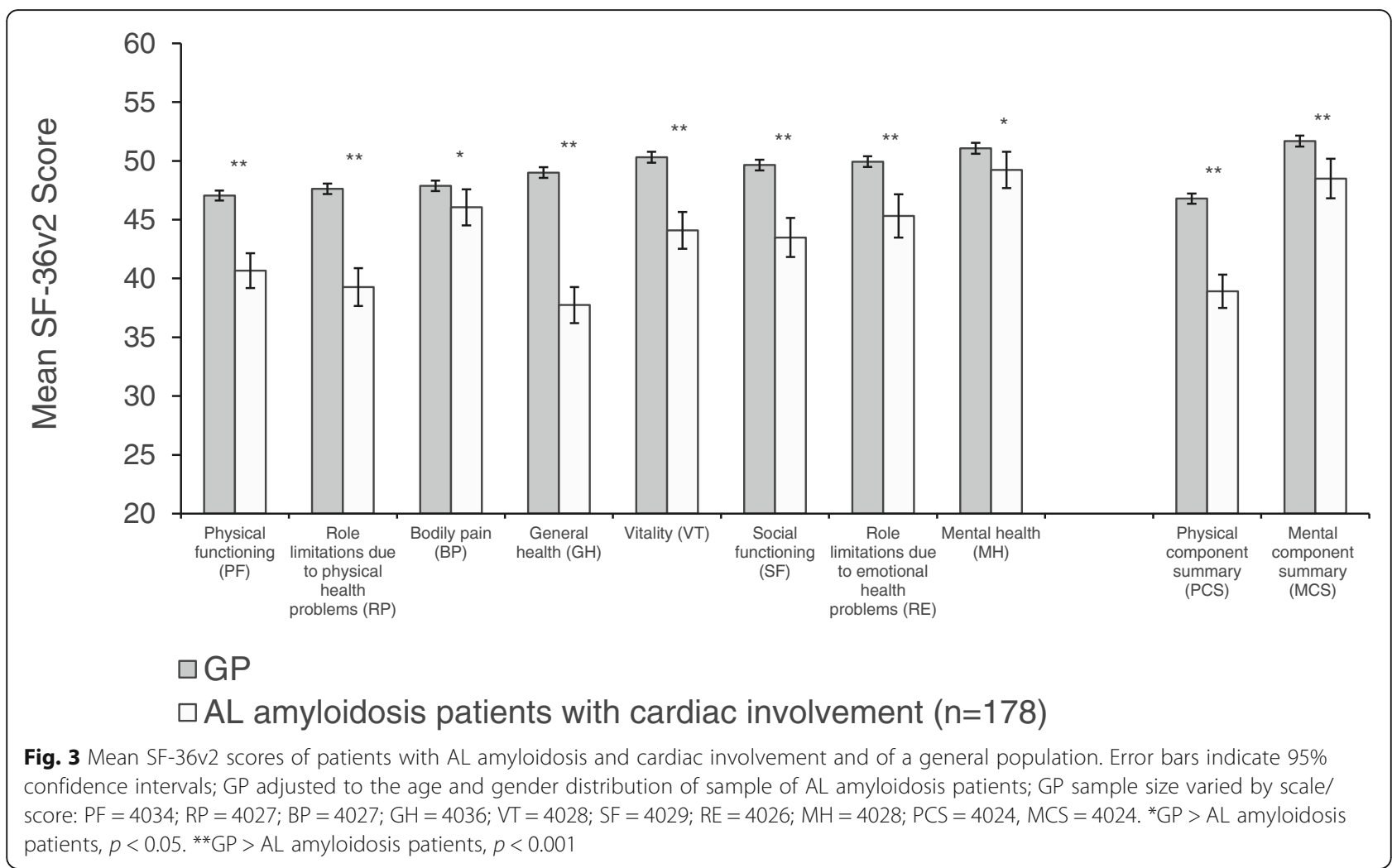

the PGI-S), other measures of disease severity were meant to be proxies for objective measures, such as HR or cardiac involvement. These proxy measures may have been affected by measurement error. HR, in particular, relies on patients' recall and understanding of information relayed to them by their clinicians. A notable proportion of the sample (11\%) acknowledged their uncertainty of their current HR status by endorsing the 'I do not know' category.

Our sample may represent a healthier subset of AL amyloidosis patients. First, despite advances in the treatment of patients with AL amyloidosis, the prevalence of sudden death within 90 days of diagnosis remains around $25-30 \%$, whereas patients who survive $>12$ months have a better prognosis [25-27]. Here, 85\% of patients in this sample received their diagnoses $\geq 12$ months ago, and the median time since diagnosis within this sample was 3.5 years. This is typical of cross-sectional studies in which short-term survivors are often underrepresented among prevalent cases [28].

Second, only the healthiest AL amyloidosis patients $(<20 \%$ of all patients) are typically eligible to undergo SCT [29], but the lifetime prevalence of SCT in this sample was $53 \%$. Finally, $41 \%$ of the sample reported current complete remission or HR, indicating that a substantial proportion of this sample responded favorably to treatment.
Despite the likelihood that this sample might have been disproportionately healthier than the broader population of AL amyloidosis patients, the demographic characteristics of our study population mirror those previously described in the literature, including those of patients with newly diagnosed AL amyloidosis and longterm survivors with a range of organ involvement $[3,14]$. However, given the likelihood that our sample represented healthier patients, it is probable that the HRQoL in patients with AL amyloidosis is actually lower.

These findings may help clinicians understand how their patients are impacted by AL amyloidosis. Understanding the burden of AL amyloidosis can help physicians identify ancillary treatments and services that may ease patients' disease burden and ultimately improve their HRQoL. Subsequently, this may improve the doctor-patient relationship through a patient-centered approach.

Furthermore, these findings may interest regulatory groups, such as the FDA and the EMA. FDA's Guidance for Industry in Rare Diseases specifically notes that rare diseases are highly diverse and that, due to the small number of patients affected and the clinical expertise dispersed among a small number of treatment centers, the natural history of rare diseases is often poorly described, as are "patient reports of function and feeling" [30]. The FDA notes that drug developers should 
conduct observational studies to facilitate understanding of the population and unmet need, to define the disease population and subtypes, and to design appropriate clinical trials with suitable outcome measures that are sensitive to change in disease status and that are reliable and valid for the intended use.

\section{Conclusions}

Overall these data demonstrated significant deficits in HRQoL among AL amyloidosis patients and an additional burden among clinically relevant subgroups of patients. Further, this study indicates that the SF-36v2 is sensitive to variations in HRQoL across groups known to differ in disease severity. The study is continuing to collect data to evaluate the change in HRQoL over time and in other subgroups. Given the importance of providing patient-centered care and including the patient's voice in research, these findings are relevant to a variety of stakeholders, among them physicians, patients, payors, and regulatory groups.

\section{Abbreviations \\ AL: Amyloid light chain; BP: Bodily pain; EMA: European medicines agency; FDA: United States food and drug administration; GED: General educational development; GH: General health; GP: General population; HR: Hematologic response; HRQoL: Health-related quality of life; ICF: Informed consent form; MCID: Minimal clinically important difference; MCS: Mental component summary; MH: Mental health; PCS: Physical component summary; PF: Physical functioning; PGI-S: Patient global impression-severity scale; RE: Role emotional; RP: Role physical; SCT: Stem cell transplantation; SD: Standard deviation; SF: Social functioning; SF-36v2: SF-36v2 health survey ${ }^{\oplus}$; VT: Vitality}

\section{Acknowledgments}

The authors thank the patients for their participation in this study and the Amyloidosis Support Group and the Amyloidosis Foundation for their help in patient outreach.

Portions of this work were presented in poster format at the 21st Congress of the European Hematology Association, June 9-12, 2016, Copenhagen, Denmark, and at the International Society of Amyloidosis 2016 Symposium, July 3-7, 2016, Uppsala, Sweden.

\section{Funding}

A full-time employee of the study sponsor, Prothena Biosciences Inc, served as an author on this manuscript. SDG contributed to the design of the study and reviewed study results. SDG reviewed and revised the manuscript critically for important intellectual content and gave final approval of the version to be published.

\section{Availability of data and material}

The data sets analyzed in the current study are available from the corresponding author on reasonable request.

\section{Authors' contributions}

$M B, K L M, M K W$, and SDG designed the study. MB, KLM, and MKW collected data and wrote the manuscript. KLM analyzed the data. All authors revised the manuscript critically for important intellectual content and gave final approval of the version to be published.

\section{Competing interests}

$M K W, M B$, and KLM are full-time employees of Optum, Inc, which publishes the SF-36v2 ${ }^{\oplus}$, and received research funding from Prothena Biosciences Inc to conduct the study. SDG is a full-time employee of the study sponsor, Prothena Biosciences Inc.
Consent for publication

Not applicable.

\section{Ethics approval and consent to participate}

The study protocol, informed consent, and measures were approved by the New England Institutional Review Board. Online recruitment strategies were used between October and December 2015, with institutional review boardapproved scripted messages posted on amyloidosis patient advocacy group Web sites and social media sites and in membership e-mails.

\section{Author details}

'Optum, 24 Albion Road, Lincoln, RI 02865, USA. ${ }^{2}$ Prothena Biosciences Inc, South San Francisco, CA, USA.

Received: 5 October 2016 Accepted: 27 December 2016

Published online: 19 January 2017

\section{References}

1. Gertz MA. Immunoglobulin light chain amyloidosis: 2013 update on diagnosis, prognosis, and treatment. Am J Hematol. 2013;88:416-25.

2. Falk RH, Comenzo RL, Skinner M. The systemic amyloidoses. N Engl J Med. 1997;337:898-909.

3. Kyle RA, Linos A, Beard CM, Linke RP, Gertz MA, O'Fallon WM, et al. Incidence and natural history of primary systemic amyloidosis in Olmsted County, Minnesota, 1950 through 1989. Blood. 1992;79:1817-22.

4. Pinney JH, Smith CJ, Taube JB, Lachmann HJ, Venner CP, Gibbs SD, et al. Systemic amyloidosis in England: an epidemiological study. $\mathrm{Br} J$ Haematol. 2013;161:525-32.

5. Muchtar E, Buadi FK, Dispenzieri A, Gertz MA. Immunoglobulin light-chain amyloidosis: from basics to new developments in diagnosis, prognosis and therapy. Acta Haematol. 2016;135:172-90.

6. Merlini G, Palladini G. Light chain amyloidosis: the heart of the problem. Haematologica. 2013;98:1492-5.

7. Merlini G, Seldin DC, Gertz MA. Amyloidosis: pathogenesis and new therapeutic options. J Clin Oncol. 2011;29:1924-33.

8. Cohen AD, Zhou P, Chou J, Teruya-Feldstein J, Reich L, Hassoun H, et al. Risk-adapted autologous stem cell transplantation with adjuvant dexamethasone +/- thalidomide for systemic light-chain amyloidosis: results of a phase II trial. Br J Haematol. 2007;139:224-33.

9. Sanchorawala V, Skinner M, Quillen K, Finn KT, Doros G, Seldin DC. Longterm outcome of patients with AL amyloidosis treated with high-dose melphalan and stem-cell transplantation. Blood. 2007;1 10:3561-3.

10. Lin HM, Seldin D, Hui A, Berg D, Dietrich CN, Flood E. The patient's perspective on the symptom and everyday life impact of AL amyloidosis. Amyloid. 2015;22:244-51.

11. White MK, Bayliss M, Finkel M, Lousada I, Guthrie S. Health-related quality of life in patients with AL amyloidosis: qualitative interviews with physicians and patients. Presented at: American Society of Hematology 57th Annual Meeting and Exposition; December 5-8, 2015. Orlando: Abstract; 2015.

12. Smorti M, Guarnieri S, Bergesio F, Perfetto F, Cappelli F. Anxiety and depression among amyloid light-chain cardiac amyloidosis patients: the role of life satisfaction. Eur J Cardiovasc Nurs. 2016;15:269-75.

13. Caccialanza R, Palladini G, Klersy C, Cereda E, Bonardi C, Cameletti B, et al. Nutritional status independently affects quality of life of patients with systemic immunoglobulin light-chain (AL) amyloidosis. Ann Hematol. 2012;913:399-406.

14. Seldin DC, Anderson JJ, Sanchorawala V, Malek K, Wright DG, Quillen K, et al. Improvement in quality of life of patients with AL amyloidosis treated with high-dose melphalan and autologous stem cell transplantation. Blood. 2004;104:1888-93.

15. Maruish ME. User's Manual for the SF-36v2 Health Survey. 3rd ed. Lincoln: QualityMetric, Inc:; 2011.

16. Lee CW, Chi KN. The standard of reporting of health-related quality of life in clinical cancer trials. J Clin Epidemiol. 2000;53:451-8.

17. Ware Jr JE. SF-36 health survey update. Spine. 2000;25:3130-9.

18. Ware Jr JE, Sherbourne CD. The MOS 36-item short-form health survey (SF-36), I: conceptual framework and item selection. Med Care. 1992;30:473-83.

19. Hendriksz CJ, Berger Kl, Lampe C, Kircher SG, Orchard PJ, Southall R, et al. Health-related quality of life in mucopolysaccharidosis: looking beyond biomedical issues. Orphanet J Rare Dis. 2016;11:119. 
20. Bosch AM, Burlina A, Cunningham A, Bettiol E, Moreau-Stucker F, Koledova E, et al. Assessment of the impact of phenylketonuria and its treatment on quality of life of patients and parents from seven European countries. Orphanet J Rare Dis. 2015;10:80.

21. Palladini G, Dispenzieri A, Gertz MA, Kumar S, Wechalekar A, Hawkins PN, et al. New criteria for response to treatment in immunoglobulin light chain amyloidosis based on free light chain measurement and cardiac biomarkers: impact on survival outcomes. J Clin Onco. 2012;30:4541-9.

22. Guy W. ECDEU Assessment Manual for Psychopharmacology. Rockville: U.S. Department of Health Education and Welfare Public Health Service Alcohol Drug Abuse and Mental Health Administration National Institute of Mental Health Psychopharmacology Research Branch; 1976.

23. Dennis JM, Thomas RK. Documentation for Human Subject Review Committees: GfK Company Information, Past External Review, Confidentiality, and Privacy Protections for Panelists. http://www.knowledgenetworks.com/ ganp/irbsupport/docs/KN\%20Confidentiality\%20and\%20Privacy\%20 Protections\%20Doc\%20for\%20IRBs.pdf. Accessed 27 Sept 2016.

24. Cox S, O'Donoghue AC, McKenna WJ, Steptoe A. Health related quality of life and psychological wellbeing in patients with hypertrophic cardiomyopathy. Heart. 1997;78:182-7.

25. Merlini G, Wechalekar AD, Palladini G. Systemic light chain amyloidosis: an update for treating physicians. Blood. 2013;121:5124-30.

26. Kumar SK, Gertz MA, Lacy MQ, Dingli D, Hayman SR, Buadi FK, et al. Recent improvements in survival in primary systemic amyloidosis and the importance of an early mortality risk score. Mayo Clin Proc. 2011;86:12-8.

27. Grogan M, Dispenzieri A. Natural history and therapy of AL cardiac amyloidosis. Heart Fail Rev. 2015;20:155-62

28. Szklo M, Nieto FJ. Epidemiology: Beyond the Basics. Gaithersburg: Aspen Publishers; 2000

29. Merlini G. Plasma cell disorder other than myeloma: treatment of amyloidosis. Presented at: American Society of Hematology 57th Annual Meeting and Exposition; December 5-8, 2015. Orlando: Education Spotlight Session; 2015.

30. Rare Diseases: Common Issues in Drug Development: Guidance for Industry. Silver Spring, MD: US Food and Drug Administration; 2015. http://www. fda.gov/downloads/Drugs/GuidanceComplianceRegulatoryInformation/ Guidances/UCM458485.pdf. Accessed 27 Sept 2016

\section{Submit your next manuscript to BioMed Central and we will help you at every step:}

- We accept pre-submission inquiries

- Our selector tool helps you to find the most relevant journal

- We provide round the clock customer support

- Convenient online submission

- Thorough peer review

- Inclusion in PubMed and all major indexing services

- Maximum visibility for your research

Submit your manuscript at www.biomedcentral.com/submit 